First publ. in: Superlattices and Microstructures 23 (1998), 3/4, pp. 747-756

\title{
What are Landauer's conduction channels in an atomic-size metallic contact?
}

\author{
E. Scheer $\dagger$, P. Joyez, M. H. Devoret, D. Esteve, C. Urbina \\ Service de Physique de l'Etat Condensé, Commissariat à l'Energie Atomique, Saclay, \\ F-91191 Gif-sur-Yvette Cedex, France
}

(Received 10 November 1997)

\begin{abstract}
Using microfabricated break junctions, we have produced $\mathrm{Al}$ and $\mathrm{Au}$ contacts consisting of a small number of atoms. These metallic quantum point contacts accomodate a small number of conduction channels. We have determined the individual transmission coefficients in contacts containing up to six conduction channels. The determination is based on a comparison of the highly nonlinear current-voltage characteristics in the superconducting state with the predictions of the theory for a single channel superconducting contact (in the case of Au contacts superconductivity is induced by means of the proximity effect). We find that in the smallest achievable contacts usually three channels contribute to the transport in the case of $\mathrm{Al}$, whereas only one does in the case of Au. These findings are consistent with the quantum chemistry point of view that the channels are determined by the valence atomic orbitals at the narrowest section of the contacts.
\end{abstract}

(c) 1998 Academic Press Limited

Key words: atomic contacts, conduction channels, superconductivity.

\section{Introduction}

The theory of electron transport pioneered by Landauer [1] treats an electronic transport experiment on a mesoscopic sample as an ordinary wave scattering experiment. Electrons are sent from a reservoir into the sample which scatters them. A fraction of the electrons is reflected back and the rest is collected by another reservoir. Each reservoir plays the role of source and detector (for a recent review, see [2]). To establish a well defined set of electronic quantum states with which to describe the scattering experiment, the theory introduces 'leads' connecting the mesoscopic sample to the reservoirs. In the leads, a finite number $N$ of independent electronic ingoing and outgoing modes propagate. These modes constitute a finite and complete basis of states for the scattering problem. The scatterer connects in principle any incoming mode with all outgoing modes. In the theory, this is represented by a transmission matrix $t$ and a reflection matrix $r$. For example, the matrix element $t_{n m}$ gives the probability amplitude for the left-incoming mode $m$ to be transmitted into the right-outgoing mode $n$. These matrices carry all the information on the scatterer and thus determine all the transport properties of the mesoscopic sample under investigation. For example, the total conductance of a mesoscopic structure is given by the celebrated Landauer formula, $G=G_{0} \mathcal{T}$, where $\mathcal{T}=\operatorname{Tr}\left\{t^{\dagger} t\right\}$ is the total transmission of the structure and $G_{0}=2 e^{2} / h$ is the conductance quantum. However, as the trace of a matrix is invariant through any unitary basis transformation, there is no preferred basis to calculate the

$\dagger$ Present address: Physikalisches Institut, Universitat Karlsruhe, Engesserstrasse 7, 76128 Karlsruhe, Germany. 
conductance. In other words, it is not necessary to know the actual eigenvalues $\tau_{i}$ of the matrix $T=t^{\dagger} t$ in order to calculate the total transmission $\mathcal{T}=\sum\left(t^{\dagger} t\right)_{n n}=\sum \tau_{i}$. Conversely, a measurement of the conductance does not provide enough information to determine the ensemble $\left\{\tau_{i}\right\}$, unless the scatterer has only one mode. In fact, not even the total number $N_{0}$ of transmission eigenmodes of the scatterer can be extracted from the knowledge of the conductance.

However, there exist transport quantities that do not depend linearly on the $\tau_{i}$ that can provide additional information on the transmission matrix. For example, the power of the shot-noise associated with the current at voltage $V$ is a measure of the second-moment of the transmission distribution $\left\{\tau_{i}\right\}[3]$ :

$$
P=2 e V G_{0} \sum \tau_{i}\left(1-\tau_{i}\right)=2 e V\left(G-G_{0} \operatorname{Tr}\left\{T^{2}\right\}\right) .
$$

A perfectly transmitted mode $\left(\tau_{i}=1\right)$ does not contribute to the noise because of electron correlations arising from the Pauli principle. A measurement of this noise power provides then a second relation between the matrix elements of $T$. However, this information is still not sufficient to determine the eigenvalues ensemble $\left\{\tau_{i}\right\}$ if the scatterer has more than two modes.

In principle, one needs to measure a number of moments equal to the total number $N_{0}$ of channels in order to determine all the transmission coefficients. One of the main messages of the present paper is that transport experiments in the superconducting state allow us to measure the full transmission distribution. However, before going into this, let us briefly comment on famous experiments on quantum point contacts (QPCs) which are interpreted in terms of the scattering theory of transport. A QPC is a constriction between two metallic reservoirs of lateral size comparable with the Fermi wavelength $\lambda_{F}$ of the electrons. We can distinguish two realizations of QPCs.

\subsection{Adiabatic quantum point contacts}

The first kind of QPCs is fabricated on two-dimensional electron gases in epitaxially grown heterostructures of semiconductors. These gases are low electronic density $\left(10^{12} / \mathrm{cm}^{2}\right)$ metals in which the Fermi wavelength of the electrons can be as large as $30 \mathrm{~nm}$. The constriction is defined and its width $W$ is adjusted by means of nanofabricated electrostatic gates which deplete locally the electron layer. Experimentally, as the width is increased continuously, the conductance increases by steps of nearly $G_{0}$ : this is the phenomenon of conductance quantization $[4,5]$. The experiments are performed on very good realizations of ideal ballistic leads and QPCs. Moreover, the connection between the leads and the sample is almost perfectly adiabatic: the eigenmodes of both the leads and the QPC are of the same nature and they are perfectly matched. For these structures the choice of a basis of laterally confined plane waves which are funnelled through the constriction is quite natural. In this language, the conductance staircase has a very simple interpretation: if the width of the constriction is much smaller than $\lambda_{F}$ the conductance of the QPC is zero, i.e. all $\tau_{i}=0$; then, as the width is increased continuously, each time $W$ reaches an integer multiple of $\lambda_{F} / 2$ a new mode starts being transmitted $\left(\tau_{i}=1\right)$. We said before that a conductance measurement does not provide information on the individual eigenvalues. However, the fact that in the experiments at least fifteen conductance plateaus are observed in a reproducible and reversible fashion undoubtedly supports the view of channels opening one by one and reaching perfect transmission. Moreover, measurements of the shot-noise of the current going through a QPC [6] have shown that the noise power at the conductance plateaus is drastically reduced with respect to its full value, thus confirming the fact that the channel transmissions are either 0 or 1 . These experiments are remarkable achievements if one considers that we are dealing with solid-state structures and thus prone to all kinds of defects, especially at the atomic scale. The main reason for their success is the long Fermi wavelength which makes the motion of conduction electrons quite insensitive to atomic defects.

\subsection{Nonadiabatic quantum point contacts}

Lately, a different realization of a QPC has attracted much interest [7-10]. Several techniques allow the 


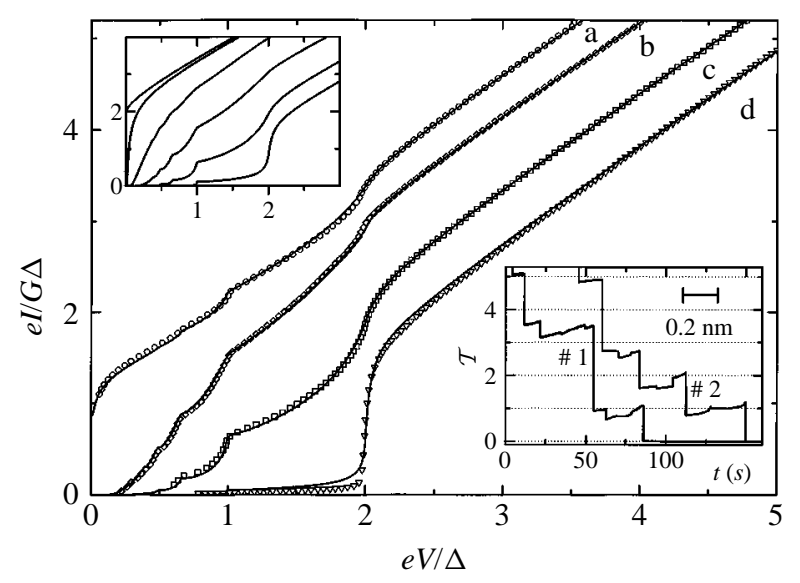

Fig. 1. Measured current-voltage characteristics (symbols) of four different configurations of sample \#1 at $30 \mathrm{mK}$ and best numerical fits (lines). The individual channel transmissions and total transmission $\mathcal{T}$ obtained from the fits are: (a) $\tau_{1}=0.997, \tau_{2}=0.46$, $\tau_{3}=0.29, \mathcal{T}=1.747$; (b) $\tau_{1}=0.74, \tau_{2}=0.11, \mathcal{T}=0.85$; (c) $\tau_{1}=0.46, \tau_{2}=0.35, \tau_{3}=0.07, \mathcal{T}=0.88$; (d) $\mathcal{T}=\tau_{1}=0.025$. Voltage and current are in reduced units. The measured superconducting gap was $\Delta / e=(182.5 \pm 2.0) \mu \mathrm{V}$. Left inset: theoretical IVs for a single channel superconducting contact for different values of its transmission coefficient $\tau$ (from bottom to top: 0.1, 0.4, 0.7, 0.9, $0.99,1)$ after [21]. Voltage and current are in reduced units. Right inset: typical total transmission traces measured at $V \geq 5 \Delta / e$, while opening the contact at around $6 \mathrm{pm} \mathrm{s}^{-1}$, for samples \#1 and \#2. The bar indicates the distance scale.

fabrication of atomic-size constrictions between two reservoirs of 'usual' metals. By usual, we mean 3D high electron density metals, like Au, Na, etc. In this case, the Fermi wavelength is comparable to the interatomic distance and the conduction electrons are sensitive to all defects at this scale. The exact geometry is not known in the experiments, but obviously there is a region around an atomic contact in which the electrons experience boundary conditions which are rough at the atomic scale. There is a 'central cluster' where many atoms are in fact surface atoms which cannot establish all their possible metallic bonds. This is precisely what limits the number of the modes that can be transmitted through the constriction. In other words, the modes of the cluster do not connect adiabatically to the modes of the reservoirs [11]. The natural language for the transport eigenmodes of the cluster is the one of linear combinations of the valence atomic orbitals [12]: The set $\left\{\tau_{i}\right\}$ of the transmission eigenvalues is thus determined both by the chemical properties of the atoms forming the contact and by their geometrical arrangement.

Experimentally, these small contacts have been obtained using both scanning tunnel microscope and breakjunction techniques $[13,14]$. The total transmission $\mathcal{T}=\sum_{i=1}^{n} \tau_{i}$ of the contacts is deduced from their measured conductance using the Landauer formula. Experiments on a large ensemble of metallic contacts have demonstrated the statistical tendency of atomic-size contacts to adopt configurations leading to some preferred values of conductance. The actual preferred values depend on the metal and on the experimental conditions. However, for many metals, and in particular 'simple' ones (like $\mathrm{Na}, \mathrm{Au}, \ldots$ ) which in bulk are good 'free electrons' metals, the smallest contacts have a conductance $G$ close to $G_{0}$ [15-17]. Does this mean that the smallest contacts (presumably a one atom contact) correspond to a single, almost perfectly transmitted channel $(\tau=1)$ ? As discussed before, this question cannot be answered solely by conductance and shot-noise measurements. We have developed instead a method that allows the determination of the full set $\left\{\tau_{i}\right\}$ [18]. This method is based on the fact that the current through superconducting constrictions builds up from a large variety of microscopic transport processes. 


\section{Transport through a superconducting QPC}

Several authors [19-21] have calculated the current-voltage characteristics $i(V, \tau)$ for a single-channel superconducting contact with arbitrary transmission $\tau$. The upper left inset of Fig. 1 shows their numerical predictions [22]. The $i(V, \tau)$ curves present a series of sharp current steps at voltage values $V=2 \Delta / n e$, where $n$ is a positive integer and $\Delta$ is the superconducting gap. Each one of these steps corresponds to a different microscopic process of charge transfer setting in. For example, the well-known nonlinearity at $e V=2 \Delta$ arises when one electronic charge $(n=1)$ is transferred thus creating two quasiparticles, one on each side of the constriction. The energy $e V$ delivered by the voltage source must be larger than the energy $2 \Delta$ needed to create the two excitations. The common phenomenon behind the other steps is multiple Andreev reflection (MAR) of quasiparticles between the two superconducting reservoirs $[23,24]$. The order $n=2,3, \ldots$, of a step corresponds to the number of electronic charges transferred in the underlying MAR process. Independently of the order, two excitations are always created. An even order process leaves both excitations in the same side of the constriction. On the contrary, odd order processes create one excitation on each side. Energy conservation $n e V=2 \Delta$ imposes the threshold for each process. For low transmission, the contribution to the current arising from the process of order $n$ scales as $\tau^{n}$. As the transmission of the channel rises from 0 to 1, the higher-order processes grow stronger and the sub-gap current increases progressively. This so-called 'subharmonic gap structure', which was in fact discovered experimentally [25], has been extensively studied in superconducting weak links and tunnel junctions with a very large number of channels [26, 27]. However, the lack of knowledge of the exact distribution $\left\{\tau_{i}\right\}$ has hindered direct quantitative comparison with the theoretical predictions. On the other hand, measurements [28] of the current-voltage characteristic (IV) of $\mathrm{Nb}$ and $\mathrm{Pb}$ single-channel tunnel junctions with an adjustable transmission $\tau_{1}$ have shown that the height of the successive current steps is proportional to increasing powers of $\tau_{1}$, in excellent agreement with the theory of MAR in the low $\tau_{1}$ regime [29]. The same series of experiments, but in the contact regime, showed qualitatively that even for a single atom contact more than one conduction channel needs to be considered.

By definition of the channels, the total current $I(V)$ through a contact characterized by the ensemble $\left\{\tau_{i}\right\}$, with $i=1, \ldots, N_{0}$ is the sum of $N_{0}$ independent terms:

$$
I(V)=\sum_{i=1}^{N_{0}} i\left(V, \tau_{i}\right)
$$

Because the probability of MAR processes depends on high powers of the transmission coefficients, the full IV curve is highly nonlinear and carries information on all the moments of the transmission distribution. In practical terms, it is possible, using numerical fits based on tabulated values of $i(V, \tau)$, to find the full distribution $\left\{\tau_{i}\right\}$ by decomposing the experimental IVs into the contribution of a finite number of channels.

\section{Determination of the channel transmissions}

\subsection{Experimental techniques}

In order to infer $\left\{\tau_{i}\right\}$ from the IV curves, very stable atomic-sized contacts are required. For this purpose we have used a microfabricated version[30] of the mechanically-controllable break-junctions pioneered by Moreland and Hansma [31] and further developed by Muller et al. [32]. Our samples are $2 \mu \mathrm{m}-\mathrm{long}, 100$ nm-thick suspended microbridges, with a $100 \mathrm{~nm} \times 100 \mathrm{~nm}$ constriction in the middle (cf. Fig. 2). The bridge is broken at the constriction by controlled bending of the elastic substrate mounted on a three-point bending mechanism. A differential screw (100 $\mu \mathrm{m}$ pitch) driven by a dc-motor through a series of reduction gear boxes, controls the motion of the pushing rod that bends the substrate (Fig. 2). The geometry of the bending mechanism is such that a $1 \mu \mathrm{m}$ displacement of the rod results in a relative motion of the two anchor 


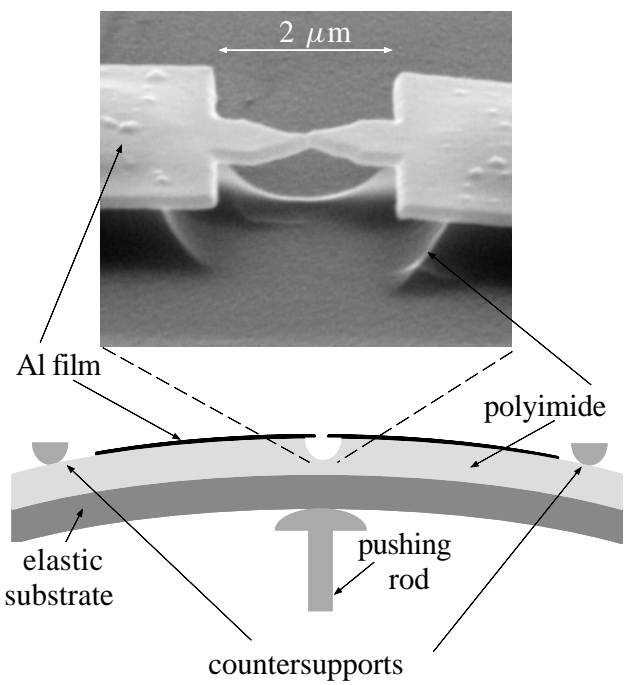

Fig. 2. Three point bending mechanism. The pushing rod bends the phosphorbronze substrate. The distance between the two countersupports was $12 \mathrm{~mm}$, and the substrate was $0.3 \mathrm{~mm}$ thick. The micrograph shows a suspended Al microbridge (sample \#2). The insulating polyimide layer was etched to free the bridge from the substrate.

points of the bridge of around $0.2 \mathrm{~nm}$. This was verified using the exponential dependence of the conductance on the interelectrode distance in the tunnel regime. This very strong dependence was used to estimate the experimental interelectrode stability to be better than $200 \mathrm{fm} /$ hour. The bending mechanism is anchored to the mixing chamber of a dilution refrigerator. The bridges are broken at low temperature $(T<1 \mathrm{~K})$ and under cryogenic vacuum (obtained with a sorption pump) to prevent contamination of the two resulting electrodes. The voltage and the current through the device are measured using two low-noise differential preamplifiers. All lines connecting the sample to the room temperature electronics are carefully filtered at microwaves frequencies by a combination of lossy shielded cables [33], and microfabricated cryogenic filters [34].

\subsection{Experimental results for Al contacts}

After breaking, the electrodes are brought back into contact to form a point contact with a resistance of a few hundred ohms. Pushing again on the substrate leads to a controlled opening of the contact, while the sample is maintained at $T<100 \mathrm{mK}$. As found in previous experiments at higher temperatures, the conductance $G$ (measured at energies well above $\Delta$ ) decreases in steps of the order of $G_{0}$, their exact sequence changing from opening to opening (see right inset of Fig. 1). Between two jumps, $G$ generally tends to increase when pulling the contact $[14,35]$. The last conductance value before the contact jumps into the tunnelling regime is usually between 0.5 and $1.5 G_{0}$. We distinguish between contact and tunnel behavior by the different interelectrode distance dependence of the conductance in both regimes. The set-up stability allows us to stop at any point along the breaking or closing curves in order to record the IVs. Figure 1 shows the IVs of four configurations obtained on sample \#1 at $T=30 \mathrm{mK}$. Note that curves (b) and (c) differ markedly even though they correspond to contacts having the same conductance within 5\%. Curve (d) was obtained after the contact broke, i.e. in the tunnel regime, and is comparable to the results reported in [28]. When the device is driven into the normal state by applying a magnetic field $B>B_{c}=10 \mathrm{mT}$, the IV becomes linear and corresponds to the same $G$ as in the superconducting state for energies well above $\Delta$. We consider this as an indication that the channels remain the same in the normal and in the superconducting states. When the magnetic field is turned off, we recover exactly the same IV as before. 


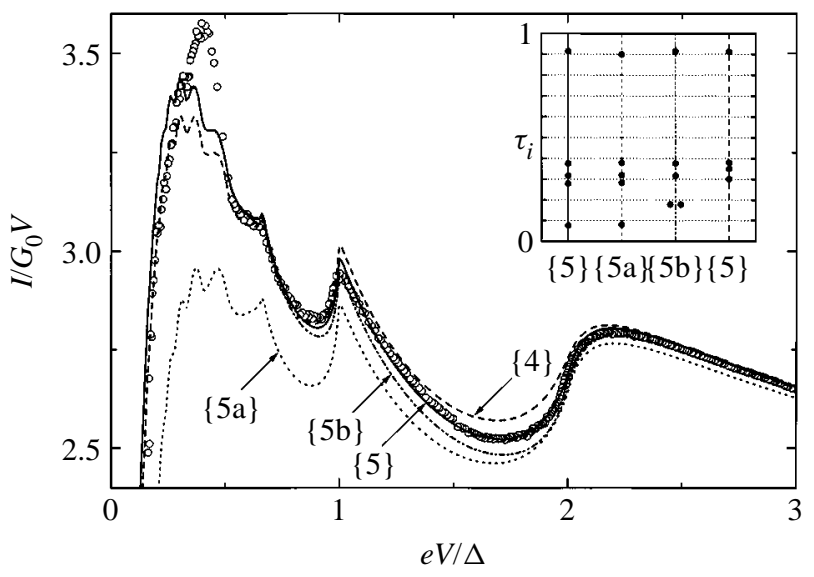

Fig. 3. Measured $(I / V)$ (circles) as a function of voltage for a contact obtained on sample \#3. We have used reduced quantities on both axis. Also shown are four calculated curves for different transmission sets $\left\{\tau_{i}\right\}:\{5\}$ five channel best fit, $\mathcal{T}=1.96 ;\{5 \mathrm{a}\}$ and $\{5 \mathrm{~b}\}$ five channel curves with slight deviations from best fit ensemble but same $\mathcal{T} ;\{4\}$ four channel best fit, $\mathcal{T}=1.94$. Inset: set $\left\{\tau_{i}\right\}$ for the four calculated curves. The set $\{5 \mathrm{a}\}$ was obtained from $\{5\}$ by reducing the transmission of the most transmitted channel by $0.75 \%$ of $\mathcal{T}$, and increasing the other 4 accordingly. The set $\{5 \mathrm{~b}\}$ was obtained from $\{5\}$ by setting the transmissions of the two less transmitted channels to their average value, keeping the 3 others unaffected. The measured gap was $\Delta / e=(185 \pm 2) \mu \mathrm{V}$. The disagreement between experimental and theoretical curves below $V=2 \Delta / 4 e$ is attributed to a resonance of the electromagnetic environment of the device [36] (see text).

In Fig. 1 we also show the best least-squares fits obtained using the numerical results of the single-channel theory [22]. The fitting procedure decomposes the total current $I(V, \mathcal{T})$ into the contributions of up to six independent channels. Channels found with transmissions lower than $1 \%$ of the total transmission are neglected. Since the voltage threshold for MAR processes decreases as the order increases, we sampled the voltage with a density factor $1 /\left(V+V_{0}\right)$ with $V_{0}=0.1 \Delta / e$. To illustrate the sensitivity of the fitting procedure, we show in Fig. $3(I / V)$ vs. $V$ for a particular contact on sample \#3 along with four curves calculated with different $\left\{\tau_{i}\right\}$. Although $\mathcal{T} \simeq 2$, a five channel fit is necessary in this case. Besides the five and four channels best fit curves we also show two curves obtained for slightly different values of the five individual transmission coefficients, keeping their sum constant (see inset of Fig. 3). When $n \leq 3$, this fitting procedure allows the determination of each $\tau_{i}$ with an accuracy of $1 \%$ of $\mathcal{T}$. For contacts containing more channels only the two or three dominant channels (depending on their absolute value) can be extracted with that accuracy: As the number of contributing channels increases, or the transmission of some individual channels approaches $\tau_{i}=1$, the information necessary to disentangle the contributions of additional channels is found in higher order MAR. Consequently, reliable data at lower voltages become increasingly important to obtain accurate fits. Experimentally, it is not possible to measure the current at arbitrarily low voltages because there is a hysteretic switching to the zero-voltage superconducting state. This sets a limitation to the maximum number of channels and the minimum value of transmission one can precisely characterize by this method. We stress that the total number of channels (with $\tau_{i}$ larger than $1 \%$ of $\mathcal{T}$ ) is perfectly determined by the fit. Only the low transmission values become less accurate when increasing $n$. In practice, a second error source affects the method: There is a residual disagreement between experiment and theory for voltages smaller than $2 \Delta / 4 e \simeq 100 \mu \mathrm{V}$ (see Fig. 3). We attribute it to inelastic Cooper pair tunnelling corresponding to the excitation of a resonant mode of the electromagnetic circuit in which the contact is embedded [36]. For the particular geometry of our samples there is a resonance at a voltage of $(60 \pm 10) \mu \mathrm{V}$ which prevents quantitative comparison below $2 \Delta / 4 e$. However, proper design of the electromagnetic environment of the 


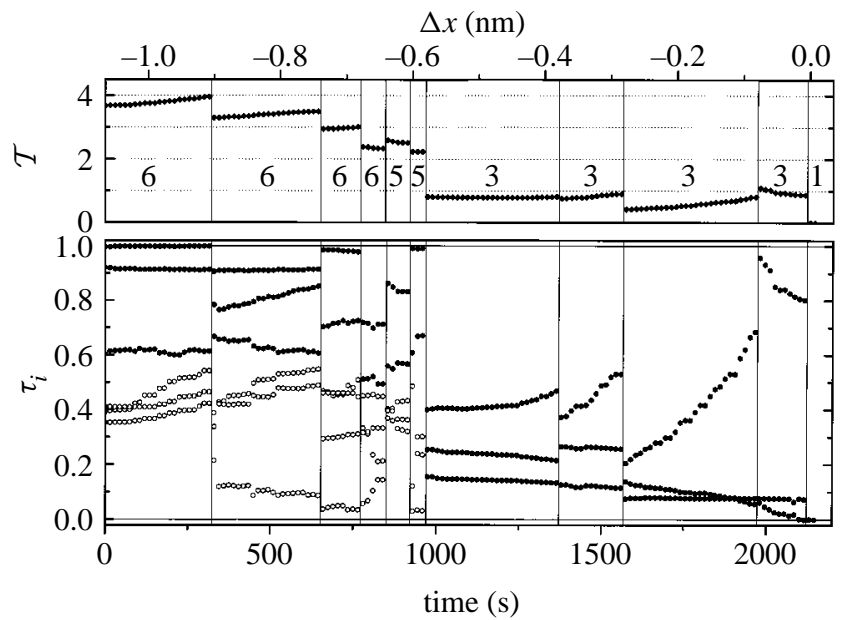

Fig. 4. Top panel: total transmission $\mathcal{T}=\sum \tau_{i}$ as a function of time, as deduced from the best fit of the IVs recorded on flight while opening sample \#3 at $0.5 \mathrm{pm} \mathrm{s}^{-1}$. Bottom panel: evolution of individual transmission coefficients $\tau_{i}$ as deduced from the fit; $(\bullet)$ channels determined with an accuracy of $1 \%$ or better of total transmission $\mathcal{T} ;(\circ)$ channels determined with an accuracy of $3 \%$ of $\mathcal{T}$ or better. The vertical lines correspond to conductance jumps. For each region we have indicated the minimum number of channels necessary to fit the data. In the last contact before the jump to the tunnel regime three channels contribute significantly to the current. The upper $\mathrm{x}$-axis scale indicates the approximate variation of the distance between anchors. The origin of the distance axis has been set to the point where the contact breaks and the transport enters the tunnel regime.

contact should eliminate this extrinsic limitation. Although here we present data on contacts for which no more than six channels contribute, the method is in principle applicable for larger contacts.

The bottom panel of Fig. 4 shows in detail the evolution of $\left\{\tau_{i}\right\}$ on sample \#3, as the contact is opened. The upper panel of Fig. 4 shows the evolution of the total transmission $\mathcal{T}$ as obtained from the sum of all individual transmissions. There are several remarkable features in this evolution. First, the abrupt changes in $\mathcal{T}$ correspond generally to a complete rearrangement of the transmission set. Second, even during the more continuous evolution on the tilted plateaus the variations of $\mathcal{T}$ arise from changes in several of the individual channels. Rubio et al. [37] proved that the jumps in conductance correspond to abrupt atomic rearrangements, and the tilted plateaus to elastic deformation of the contact. We can now extend their conclusions to the transmission of the individual conductance channels: The $\tau_{i}$ are fully determined by the atomic configuration. Third, the number of contributing channels does not always change when the total conductance changes abruptly. As shown in Fig. 4, some of the rearrangements of $\left\{\tau_{i}\right\}$ do not involve the appearance (or the disappearance) of channels. The number of channels sequence shown in Fig. 4 is not universal: in particular, contacts with two or four active channels can also be found. Fourth, more than one channel contributes to the transport even for contacts with a total conductance lower than $G_{0}$. This is a general feature: once in the contact regime, we never find $\mathrm{Al}$ contacts that can be described by a single channel, even when $G<G_{0}$. The reason for this lies on the atomic nature of the conduction channels.

\section{Discussion and conclusions}

In the case of $\mathrm{Al}$ the conduction bands result from the overlap from site to site of four orbitals: a single $3 \mathrm{~s}$ orbital and three $3 p$ orbitals. Each atom (with a $3 s^{2} 3 p^{1}$ configuration when isolated) contributes three electrons to these bands. One can thus expect up to four transmitting channels in a single atom contact. Cuevas et al. [12] have recently calculated the transmission coefficients using a tight-binding Hamiltonian in the case of some simple arrangements. In particular, they have predicted that in a single atom contact between two perfect 
tips grown along the (111) direction of a close-packed fcc structure, three modes contribute actually to the current. The symmetric combination of the $\mathrm{s}$ and $\mathrm{p}_{z}$ orbitals forms the dominant channel $\left(\tau_{1} \simeq 0.7\right)$ (the $z$ axis corresponds to the direction along the constriction). The $\mathrm{p}_{x}$ and the $\mathrm{p}_{y}$ orbitals lead to two degenerate channels $\left(\tau_{2}=\tau_{3} \simeq 0.1\right)$. Finally, the antisymmetric combination of the $\mathrm{s}$ and $\mathrm{p}_{z}$ orbitals results in a channel almost completely closed $\left(\tau_{4} \leq 10^{-4}\right)$. Although the exact transmission values depend on the structure around the central atom the main prediction of the model, namely that three channels contribute to the current through a single $\mathrm{Al}$ atom, is robust against disorder. However, the degeneracy of the $\mathrm{p}_{x}$ and the $\mathrm{p}_{y}$ modes is lifted by asymmetries in the structure. These predictions are consistent with our experimental results shown in Fig. 4. The bottom panel of Fig. 4 shows that in the last stages of the elongation the contact corresponds to three nondegenerate channels (region $-0.6 \leq \Delta x \leq 0 \mathrm{~nm}$ ). We think that all along this region the contact consists of a single atom, but that the local environment is quite disordered. There is only one point (near $\Delta x \approx-0.1$ $\mathrm{nm}$ ) where the actual transmission values correspond to those predicted for the perfectly ordered tips.

The method can be applied to other superconductors. Indeed, results on $\mathrm{Nb}$ [38] and $\mathrm{Pb}$ [39] confirm this 'quantum chemistry' view of transport. Five channels contribute to the current through the smallest $\mathrm{Nb}$ contacts (isolated atom configuration $4 \mathrm{~d}^{4} 5 \mathrm{~s}^{1}$ ). Three to four channels are present in the case of $\mathrm{Pb}$ (atom configuration $\left.6 \mathrm{~s}^{2} 6 \mathrm{p}^{2}\right)$.

We have also been able to test these ideas in the case of Au contacts (atomic configuration $6 \mathrm{~s}^{1}$ ) forced in the superconducting state by the proximity effect. We find that the smallest contacts correspond to a single channel, as expected from the single valence orbital for a single atom contact. Let us mention that in the four samples which we have measured up to now the conductance staircase obtained as the contact is stretched displays many steps smaller than $G_{0}$, and that the transmission of single channel contacts are distributed between 0.2 and 1. We interpret these results as the result of strong disorder in the central cluster[40].

It is interesting to note that Stafford et al. [41], using a jellium model which does not take into account the discreteness of the ionic background, arrived to the conclusion that 'conductance channels act as delocalized metallic bonds'. In the quantum chemistry point of view presented here, the delocalized metallic bonds are the conductance channels.

In conclusion, we have demonstrated that the full set of transmission coefficients in an atomic-sized contact is amenable to measurement. This is achieved by decomposing the total measured current going through the contact in the superconducting state, into the contributions of a small number of independent channels. The individual contributions are calculated using the theory for superconducting single channel contacts [19-21] valid for all values of the transmission coefficient. On the one hand, the fact that it is possible to fit with a small number of parameters the highly nonlinear energy dependence of the current for a large number of different contacts made of different materials constitutes a strong test of validity for the theory. On the other hand, the great quality of the fits allows a precise determination of the actual number of channels and their transmission. In the particular case of $\mathrm{Al}$, at least two and, more frequently, three conduction channels contribute to the current in the smallest contacts. In the case of $\mathrm{Au}$, the smallest contacts accommodate only one channel. The ensemble of the results on different materials is consistent with both the idea that the smallest contacts do consist of a single atom and the 'chemist' view that the number of channels is determined by the atomic configuration of the element. The conduction channels in atomic-size metallic contacts correspond to the conduction bands constructed from the localized atomic orbitals [12].

Acknowledgements-We thank N. Agraït and G. Rubio, B. Ludoph and J. M. van Ruitenbeek, and J. C. Cuevas, A. Martín Rodero and A. Levy Yeyati for communicating us their results prior to publication. We would also like to thank them for lively discussions from which the quantum chemistry picture of conduction channels presented here has emerged. We thank Norman O. Birge, S. Guéron and H. Pothier for their critical reading of our manuscript. This work was partially supported by the Bureau National de la Métrologie. E. Scheer acknowledges financial support by the Deutsche Forschungsgemeinschaft and CEA. 


\section{References}

[1] R. Landauer, IBM J. Res. Dev. 1, 223 (1957); Philos. M. 21, 863 (1970).

[2] C. W. J. Beenakker and H. van Houten, Solid State Phys. 44, 1 (1991); C. W. J. Beenakker, Rev. Mod. Phys. 69, 731 (1997).

[3] M. Büttiker, Phys. Rev. Lett. 65, 2901 (1990).

[4] B. J. van Wees et al., Phys. Rev. Lett. 21, 848 (1988)

[5] D. A. Wharam et al., J. Phys. C21, L209 (1988).

[6] A. Kumar, L. Saminadayar, and D. C. Glattli, Phys. Rev. Lett. 76, 2778 (1996).

[7] N. D. Lang, Phys. Rev. B52, 5335 (1995).

[8] C. Sirvent, J. G. Rodrigo, S. Vieira, L. Jurczyszyn, N. Mingo, and F. Flores, Phys. Rev. B53, 16086 (1996).

[9] T. N. Todorov and A. P. Sutton, Phys. Rev. Lett. 70, 2138 (1993); A. M. Bratkovsky, A. P. Sutton, and T. N. Todorov, Phys. Rev. B52, 5036 (1995).

[10] Uzi Landman, W. D. Luedtke, Brian E. Salisbury, and Robert L. Whetten, Phys. Rev. Lett. 77, 1362 (1996); R. N. Barnett and Uzi Landman, Nature 387, 788 (1997).

[11] Mads Brandbyge, Mads R. Sørensen, and Karsten W. Jacobsen, Phys. Rev. B56, 14956 (1997).

[12] J. C. Cuevas, A. Martín-Rodero, and A. Levy Yeyati, to be published in Phys. Rev. Lett.

[13] N. Agraït, J. C. Rodrigo, and S. Vieira, Phys. Rev. B47, 12345 (1993); C. Untiedt, G. Rubio, S. Vieira, and N. Agraït, Phys. Rev. B56, 2154 (1997).

[14] J. M. Krans, C. J. Muller, I. K. Yanson, Th. M. Govaert, R. Hesper, and J. M. van Ruitenbeek, Phys. Rev. B48, 14271 (1993) and references therein.

[15] L. Olesen, E. Lægsgaard, I. Stensgaard, F. Besenbacher, J. Schiøtz, P. Stolze, K. W. Jacobsen, and J. K. Nørskov, Phys. Rev. Lett. 72, 2251 (1994).

[16] J. M. Krans, J. M. van Ruitenbeek, V. V. Fisun, I. K. Yanson, and L. J. de Jongh, Nature 375, 767 (1995).

[17] J. L. Costa-Krämer, N. García, P. García-Mochales, and P. A. Serena, Surface Science 342, L1144 (1995).

[18] E. Scheer, P. Joyez, D. Esteve, C. Urbina and M. H. Devoret, Phys. Rev. Lett. 78, 3535 (1997).

[19] L. B. Arnold, Journal of Low Temp. Phys. 68, 1 (1987).

[20] D. Averin and A. Bardas, Phys. Rev. Lett. 75, 1831 (1995).

[21] J. C. Cuevas, A. Martín-Rodero, and A. Levy Yeyati, Phys. Rev. B54, 7366 (1996).

[22] The three works [18-20] deal through different approaches with the same physics and provide essentially the same results for the IV. We have used the numerical results provided by [21] in order to draw the inset of Fig. 1 and to perform the fits.

[23] T. M. Klapwijk, G. E. Blonder, and M. Tinkham, Physica 109,110B, 1657 (1982).

[24] M. Hurd, S. Datta, and P. F. Bagwell, Phys. Rev. B54, 6557 (1996).

[25] B. N. Taylor and E. Burstein, Phys. Rev. Lett. 10, 14 (1963).

[26] K. Flensberg and J. Bindslev Hansen, Phys. Rev B40, 8693 (1989).

[27] A. W. Kleinsasser, R. E. Miller, W. H. Malison, and G. B. Arnold, Phys. Rev. Lett. 72, 1738 (1994).

[28] N. van der Post, E. T. Peters, I. K. Yanson, and J. M. van Ruitenbeek, Phys. Rev. Lett. 73, 2611 (1994).

[29] E. N. Bratus, V. S. Shumeiko, and G. Wendin, Phys. Rev. Lett. 74, 2110 (1995).

[30] J. M. van Ruitenbeek, A. Alvarez, I. Piñeyro, C. Grahmann, P. Joyez, M. H. Devoret, D. Esteve, and C. Urbina, Rev. Sci. Inst. 67, 108 (1996).

[31] J. Moreland and P. K. Hansma, Rev. Sci. Inst. 55, 399 (1984).

[32] C. J. Muller, J. M. van Ruitenbeek, and L. J. de Jongh, Physica C191, 485 (1992).

[33] D. C. Glattli, P. Jacques, A. Kumar, P. Pari, and L. Saminadayar, J. Appl. Phys. 81, 7350 (1997).

[34] D. Vion, P. F. Orfila, P. Joyez, D. Esteve, and M. H. Devoret, J. Appl. Phys. 77, 2519 (1995).

[35] Daniel Sánchez-Portal, Carlos Untiedt, José M. Soler, Juan J. Sáenz, and Nicolás Agrait, to appear in Phys. Rev. Lett.

[36] T. Holst, D. Esteve, C. Urbina, and M. H. Devoret, Phys. Rev. Lett. 73, 3455 (1994). 
[37] G. Rubio, N. Agraït, and S. Vieira, Phys. Rev. Lett. 76, 2302 (1996).

[38] B. Ludoph and J. M. van Ruitenbeek, private communication (1997).

[39] G. Rubio and N. Agrait, private communication (1997).

[40] P. García-Mochales, P. A. Serena, N. García, and J. L. Costa-Krämer, Phys. Rev. B53, 1 (1996); P. GarcíaMochales and P. A. Serena, Phys. Rev. Lett. 79, 2316 (1997).

[41] C. A. Stafford, D. Baeriswyl, and J. Bürki, Phys. Rev. Lett. 79, 2863 (1997). 